EPJ Web of Conferences 38, 04002 (2012)

DOI: $10.1051 /$ epjconf/20123804002

(c) Owned by the authors, published by EDP Sciences, 2012

\title{
The first quadrupole excitations in spherical nuclei and nuclear pairing
}

\author{
S. V. Tolokonnikov ${ }^{1,2}$, S. Kamerdzhiev ${ }^{3}$, S. Krewald ${ }^{4}$, E. E. Saperstein ${ }^{1, a}$, and D. Voitenkov ${ }^{3}$ \\ 1 Kurchatov Institute, 123182, Moscow, Russia \\ 2 Moscow Institute of Physics and Technology, 123098 Moscow, Russia \\ 3 Institute for Physics and Power Engineering, 249033 Obninsk, Russia \\ 4 Institut für Kernphysik, Forschungszentrum Jülich, D-52425 Jülich, Germany
}

\begin{abstract}
Excitation energies and transition probabilities of the first $2^{+}$excitations in even lead, tin and nickel isotopes are calculated within the self-consistent Theory of Finite Fermi Systems based on the Energy Density Functional by Fayans et al. A reasonable agreement with available experimental data is obtained. The effect of the density dependence of the effective pairing interaction is analyzed in detail by comparing results obtained with volume and surface pairing. The effect is found to be noticeable, especially for the $2^{+}$-energies which are systematically higher at 200-300 keV for the volume paring as compared with the surface pairing case, the latter being in a better agreement with the data.
\end{abstract}

\section{Introduction}

Since the famous paper of 1960 by Belyaev [1], it became clear that the low-energy nuclear dynamics is governed mainly with an interplay of pairing and the quadrupole degree of freedom, properties of the quadrupole excitations themselves depending crucially on the pairing. It is clearly seen directly from the experimental data. Indeed, there is no low-lying collective $2^{+}$-state in magic nuclei where pairing is absent. E.g., in the ${ }^{208} \mathrm{~Pb}$ nucleus the first $2^{+}$-state has the excitation energy $\omega_{2}=4.08 \mathrm{MeV}$, but in the neighboring isotope ${ }^{206} \mathrm{~Pb}$ it decreases to $\omega_{2}=0.80 \mathrm{MeV}$. It is better to say that the low-lying $2^{+}$-states and pairing have the common grounds, the unclosed shell structure where low energy virtual transitions of positive parity dominate, and pairing is just an approximate way to take into account the most important part of these correlations. In the paper [2], we put attention to effects of the density dependence of pairing on characteristics of $2^{+}$-states.

Almost 50 years ago, in Ref. [3], the density-dependent ansatz for the effective pairing interaction was proposed within the theory of finite Fermi systems (TFFS) [4] with two (dimensionless) parameters $\gamma_{\mathrm{in}}$ and $\gamma_{\mathrm{ex}}$ which determine the strength of pairing interaction inside and outside nucleus correspondingly. Arguments in favor of the version with the surface dominance, $\left|\gamma_{\text {in }} / \gamma_{\mathrm{ex}}\right| \simeq 10$, were found in [3] and later in [5] on the base of the detailed analysis of double odd-even mass differences in spherical nuclei. For brevity, we name it the "surface pairing", in contrast with the "volume pairing" model corresponding to equality $\gamma_{\mathrm{in}}=\gamma_{\mathrm{ex}}$. Very strong support of the surface version was produced by Fayans et al. [6] with the analysis, within the Energy Density Functional (EDF) method, of the odd-even staggering effect in charge radii of many isotopic chains. The surface pairing version is supported also with the microscopic theory of pairing [7] starting from a realistic $N N$ potential. Here, following to [2], we demon- strate that the systematic, partially new, data on the excitation energies $\omega_{2}$ in semi-magic nuclei evidence also in favor of the surface pairing.

\section{Brief formalism}

We describe the quadrupole excitations in spherical nuclei within the self-consistent TFFS [8] with the use of the EDF method of [6]. In this method, the ground state energy of a nucleus is considered as a functional of normal and anomalous densities,

$$
E_{0}=\int \mathcal{E}\left[\rho_{n}(\mathbf{r}), \rho_{p}(\mathbf{r}), v_{n}(\mathbf{r}), v_{p}(\mathbf{r})\right] d^{3} r
$$

This approach is a generalization for superfluid finite systems of the original Kohn-Sham EDF method [9]. The normal part of the EDF $\mathcal{E}_{\text {norm }}$ contains the finite-range central, spin-orbit and effective tensor nuclear terms and Coulomb interaction term for protons. We use the DF3-a functional [10] which is a slight modification of the DF3 functional of [6] concerning the spin-orbit and effective tensor terms.

Within the TFFS, the response of a nucleus to the external quadrupole field $V_{0} \exp (i \omega t)$ can be found in terms of the effective field. In systems with pairing correlations, equation for the effective field can be written in a compact form as

$$
\hat{V}(\omega)=\hat{V}_{0}(\omega)+\hat{\mathcal{F}} \hat{A}(\omega) \hat{V}(\omega),
$$

where all the terms are matrices. In the standard TFFS notation [4], we have

$$
\begin{gathered}
\hat{V}=\left(\begin{array}{c}
V \\
d_{1} \\
d_{2}
\end{array}\right), \quad \hat{V}_{0}=\left(\begin{array}{c}
V_{0} \\
0 \\
0
\end{array}\right), \\
\hat{\mathcal{F}}=\left(\begin{array}{ccc}
\mathcal{F} & \mathcal{F}^{\omega \xi} & \mathcal{F}^{\omega \xi} \\
\mathcal{F} \xi \omega & \mathcal{F}^{\xi} & \mathcal{F}^{\xi \omega} \\
\mathcal{F} \xi \omega & \mathcal{F}^{\xi \omega} & \mathcal{F}^{\xi}
\end{array}\right),
\end{gathered}
$$

${ }^{a}$ e-mail: saper@mbslab.kiae.ru 


$$
\hat{A}(\omega)=\left(\begin{array}{ccc}
\mathcal{L}(\omega) & \mathcal{M}_{1}(\omega) & \mathcal{M}_{2}(\omega) \\
O(\omega) & -\mathcal{N}_{1}(\omega) & \mathcal{N}_{2}(\omega) \\
O(-\omega) & -\mathcal{N}_{1}(-\omega) & \mathcal{N}_{2}(-\omega)
\end{array}\right),
$$

where $\mathcal{L}, \mathcal{M}_{1}$, and so on stand for integrals over $\varepsilon$ of the products of different combinations of two Green functions $G(\varepsilon)$ and two Gor'kov functios $F^{(1)}(\varepsilon)$ and $F^{(2)}(\varepsilon)$. They can be found in [4] and we write down here only the first of them which is of the main importance for us,

$$
\mathcal{L}=\int \frac{d \varepsilon}{2 \pi i}\left[G(\varepsilon) G(\varepsilon+\omega)-F^{(1)}(\varepsilon) F^{(2)}(\varepsilon+\omega)\right] .
$$

Isotopic indices in Eqs. (3-6) are omitted for brevity. In Eq. (4), $\mathcal{F}$ is the usual Landau-Migdal amplitude,

$$
\mathcal{F}=\frac{\delta^{2} \mathcal{E}}{\delta \rho^{2}},
$$

$\mathcal{F}^{\xi}$ is the effective pairing interaction,

$$
\mathcal{F}^{\xi}=\frac{\delta^{2} \mathcal{E}}{\delta v^{2}},
$$

whereas the amplitudes $\mathcal{F}^{\omega \xi}=\mathcal{F}^{\xi \omega}$ stand for the mixed second derivatives,

$$
\mathcal{F}^{\omega \xi}=\frac{\delta^{2} \mathcal{E}}{\delta \rho \delta v}
$$

In the case of volume pairing, we have $\mathcal{F}^{\omega \xi}=0$. The explicit form of the above equations is written down for the case of the electric ( $t$-even) symmetry we deal with. A static moment of an odd nucleus can be found in terms of the diagonal matrix element $\left\langle\lambda_{0}|V(\omega=0)| \lambda_{0}\right\rangle$ of the effective field over the state $\lambda_{0}$ of the odd nucleon.

The effective field operator $\hat{V}(\omega)$ has a pole in the excitation energy $\omega_{s}$ of the state $|s\rangle$ under consideration,

$$
\hat{V}(\omega)=\frac{\left(\hat{V}_{0} \hat{A}\left(\omega_{s}\right) \hat{g}_{0 s}\right) \hat{g}_{0 s}}{\omega-\omega_{s}}+\hat{V}_{R}(\omega) .
$$

The quantity $\hat{g}_{0 s}$ has the meaning of the corresponding excitation amplitude. It obeys the homogeneous counterpart of Eq. (2) and is normalized as follows [4],

$$
\left(\hat{g}_{0 s}^{+} \frac{d \hat{A}}{d \omega} \hat{g}_{0 s}\right)_{\omega=\omega_{s}}=-1,
$$

with obvious notation.

It should be noted that the above TFFS equations are essentially equivalent to QRPA. Important difference occur if one goes beyond the QRPA [11]. For the pairing, we use the diagonal approximation, $\Delta_{\lambda \lambda^{\prime}}=\Delta_{\lambda} \delta_{\lambda \lambda^{\prime}}$, and "developed pairing" formalism which neglects effects of particle non-conservation. According to [12], it leads to errors $\simeq 0.1 \mathrm{MeV}$ in values of $\Delta_{\lambda}$.

\section{Surface versus volume pairing for semi-magic nuclei}

The QRPA-like equations of the self-consistent TFFS were solved in the coordinate representation, with exact consideration of continuum. We limit ourselves with semi-magic

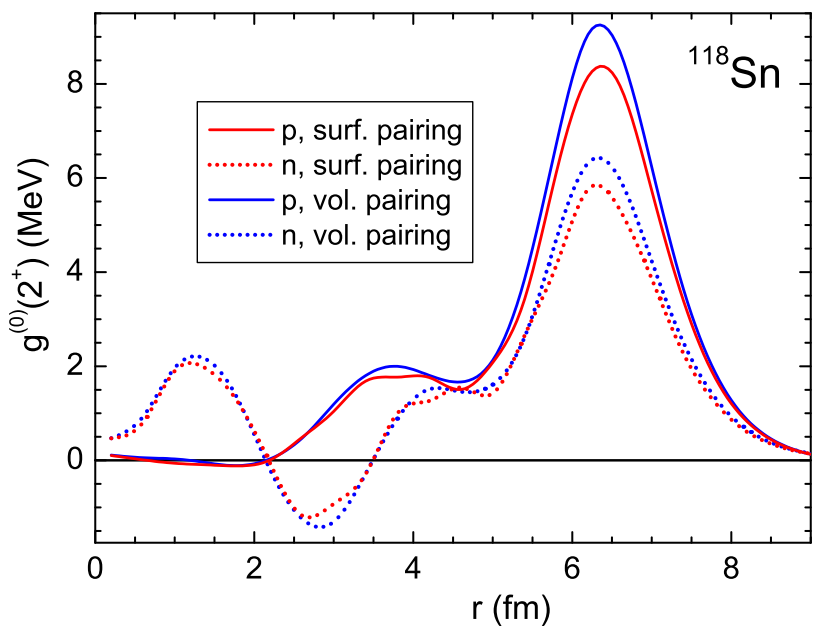

Fig. 1. The proton and neutron normal transition amplitudes $g^{(0)}$ in ${ }^{118} \mathrm{Sn}$ nucleus.

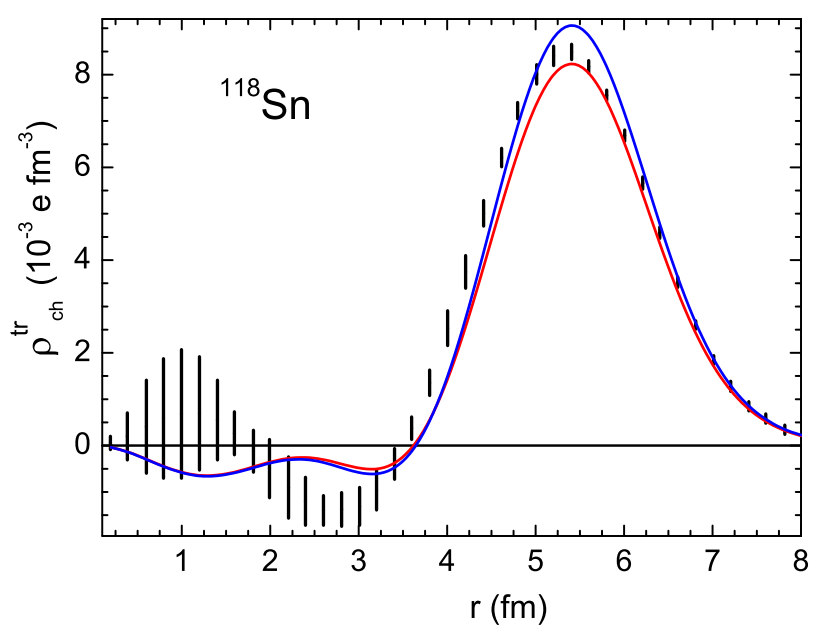

Fig. 2. The charge transition densities $\rho_{\mathrm{ch}}^{\mathrm{tr}(0)}$ in ${ }^{118} \mathrm{Sn}$ nucleus. Red line corresponds to surface pairing, blue one, to volume pairing. Experimental data are taken from [13].

nuclei where the phonon coupling corrections to QRPA are less than in nuclei with both non-magic subsystems. As a benchmark nucleus, we take ${ }^{118} \mathrm{Sn}$ which is in the middle of the tin chain. Let us begin from the normal components. As it is seen from figure 1, the normal transition amplitudes $g^{(0)}(r)$ found for these two kinds of pairing are rather close to each other. The same is true for the normal components of transition densities that is illustrated for the charge transition density $\rho_{\mathrm{ch}}^{\operatorname{tr}(0)}(r)$ which is compared in figure 2 with the experimental one found in Ref. [13] with the modelindependent analysis of the high-precision data on the elastic electron scattering. It is worth mentioning that these two figures confirm the interpretation by Khodel $[14,8]$ of the $2_{1}^{+}$-excitations as quantum capillary waves belonging to the Goldstone branch of surface vibrations.

Another situation appears for the anomalous transition amplitudes $g^{(1,2)}$, see figure 3 . We see that now these components in the case of the surface pairing are approximately 3 times stronger than for volume pairing. Unfortunately, there is no direct way to observe the anomalous transition amplitudes $g^{(1,2)}$, but there is an indirect one. When comparing figures 1 and 3, one should take into account that 


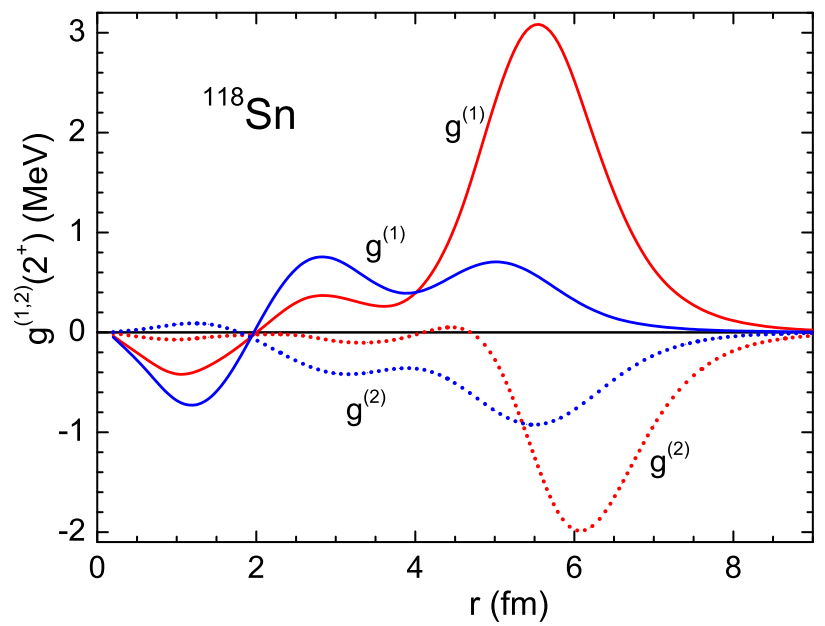

Fig. 3. The neutron anomalous transition amplitudes $g^{(1,2)}$ in ${ }^{118} \mathrm{Sn}$ nucleus. Red solid and dotted lines correspond to surface pairing, blue ones, to volume pairing.

the amplitudes $g^{(0)}$ and $g^{(1,2)}$ have the same normalization, therefore their absolute values may be compared directly. Thus, we see that in the nucleus under consideration the anomalous components are comparatively small, approximately 3 times smaller than the normal one even for the surface pairing case. Therefore we can consider the anomalous components as a small perturbation and present the eigen-energy of the $L$-phonon as

$$
\omega_{L}=\omega_{L}^{(0)}+\delta \omega_{L}^{(1)}+\delta \omega_{L}^{(2)},
$$

where the corrections due to the anomalous components are

$$
\delta \omega_{L}^{(1,2)} \propto-\left(g^{(1,2)}\right)^{2} / \omega_{L} .
$$

Here $g^{(1,2)}$ denote average values of the corresponding matrix elements. Therefore, for the surface pairing this (negative) correction should be larger. It turns out that way in actual calculations. For $\mathrm{B}(E 2)$ values, there is an interference between the normal amplitude $g^{(0)}$ and the anomalous ones, $g^{(1,2)}$, therefore dependence of the result on the type of pairing is not so definite.

Calculation results for $\omega_{2}$ values in tin isotopes are displayed in figure 4 . We see that for the surface pairing $\omega_{2}$ values are approximately by $0.3 \mathrm{MeV}$ less than for the volume one, in a qualitative accordance with Eq. (13). The surface version turns out to be closer to the experiment. The rms deviation of the theory from the experiment is $\Delta \omega_{\text {rms }}=0.16 \mathrm{MeV}$ for the surface pairing and $\Delta \omega_{\text {rms }}=0.37$ $\mathrm{MeV}$ for the volume pairing. When calculating the rms values of $\Delta \omega_{\text {rms }}$, we excluded the double magic nuclei and their neighbors, ${ }^{102} \mathrm{Sn}$ and ${ }^{130-134} \mathrm{Sn}$. Magic nuclei are excluded on the reason of absence of pairing and hence the effect under consideration. As to their neighbors, the reason is deficiencies of the "developed pairing" approximation we use for such nuclei. First, the particle number conservation only in average inherent to this approximation works for them rather poorly, as it follows from the analysis in Ref. [12]. Second, it is true for the equality of the operators $\Delta^{+}$and $\Delta^{-}$, see Ref. [4], which is supposed in this approximate scheme. Indeed, e.g. for an isotope with the neutron number $N=N_{\text {mag }}-2$, the operator $\Delta^{+}$connects the nucleus under consideration with the one in which the neutron pairing is absent.

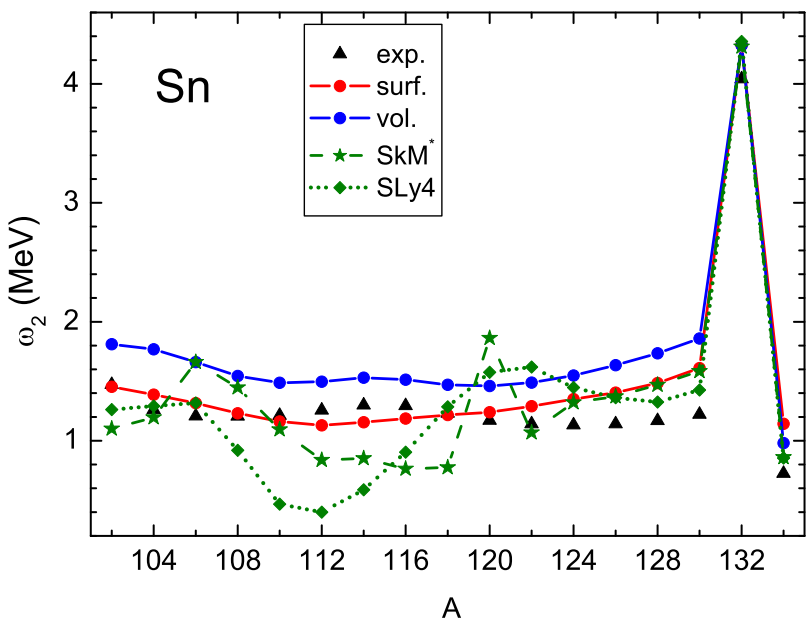

Fig. 4. Excitation energies $\omega\left(2_{1}^{+}\right)$for tin isotopes. Experimental data are taken from [15].

The $\mathrm{B}(E 2)$ values in the tin chain are shown in figure 5 . In this case, the situation is not so definite because of experimental errors. It is worth mentioning that in this case the volume version looked a bit better when the old data were used [2], but new data of [16] agree better with the surface version. A comparison is made with the SkyrmeHartree-Fock-Bogolyubov (SHFB) theory predictions of Ref. [17] with the SkM* and SLy4 Skyrme force. Among these two SHFB results, the SkM* ones are significantly better. Our results for the excitation energy, especially for the surface pairing, are much better than both the SHFB ones. The same is true for the $\mathrm{B}(E 2)$ values. A general deficiency of the two SHFB theory predictions is their nonregular $A$-dependence whereas the data depend on $A$ rather smoothly.

For the lead isotopes, the situation is qualitatively similar, see figures 6, 7. Again, $\omega_{2}$ values for the volume pairing are at $0.3 \mathrm{MeV}$ higher of those for the surface case. Now we have $\Delta \omega_{\mathrm{rms}}=0.33 \mathrm{MeV}$ for surface pairing and $\Delta \omega_{\mathrm{rms}}=0.47 \mathrm{MeV}$ for volume pairing. In accordance with the above arguments, the calculation of the $\Delta \omega_{\text {rms }}$ values is carried out with exclusion of ${ }^{206-210} \mathrm{~Pb}$ nuclei. We see that here accuracy of our calculations is worse than for the tin chain but again the surface pairing model predictions look better. Here the SHFB results for both the versions of Skyrme force look much better than for the tin chain and their accuracy is approximately the same as ours for the surface pairing case. The $\mathrm{B}(E 2)$ values are known only for four lead isotopes, ${ }^{204-210} \mathrm{~Pb}$, the SHFB accuracy is a bit higher. Note that the ${ }^{204} \mathrm{~Pb}$ nucleus is the only among them which is described within our QRPA-like scheme adequately.

Let us go to the nickel isotopes. Figure 8 demonstrates that the two versions of the pairing force we compare are practically equivalent to each other from the point of view of reproducing the data on the neutron separation energy $S_{n}$ for the nickel chain. Analogous demonstration for the tin and lead isotopes can be found in [2]. Calculation results for excitations energies are displayed in figure 9 and for $\mathrm{B}(E 2)$ values, in figure 10 . The chain contains two double-magic nuclei, ${ }^{56} \mathrm{Ni}, N=28$, and ${ }^{78} \mathrm{Ni}, N=50$. In addition, the nucleus ${ }^{68} \mathrm{Ni}$ with the neutron number $N=40$ also can be interpreted as a quasi-magic one. Indeed, the excitation energy of the $2_{1}^{+}$state in this nucleus is signifi- 


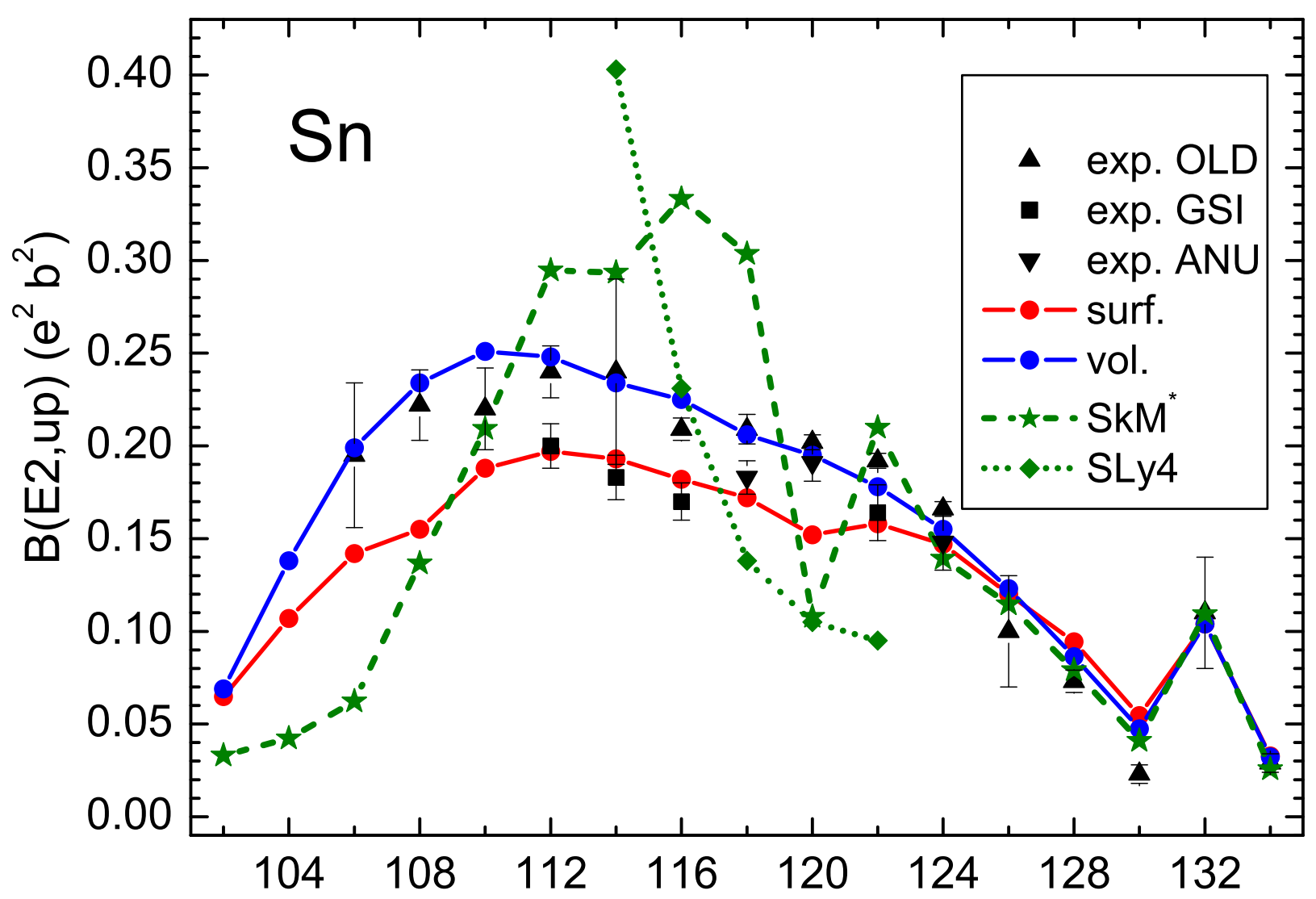

A

Fig. 5. $B\left(E 2\right.$, up) values for tin isotopes. The "old" experimental data are taken for ${ }^{114-124}$ Sn from [15], for ${ }^{126-134} \mathrm{Sn}$ from [18], and for ${ }^{106-112} \mathrm{Sn}$ from [19-21]. The new values marked "GSI" and "ANU" are taken from [16]. The first values are measured at the at the UNILAC accelerator of the Gesellschaft für Schwerionenforschung (GSI). The second ones are found also in [16] by analyzing data obtained in the magnetic moment measurement [22] at the Australian National University (ANU).

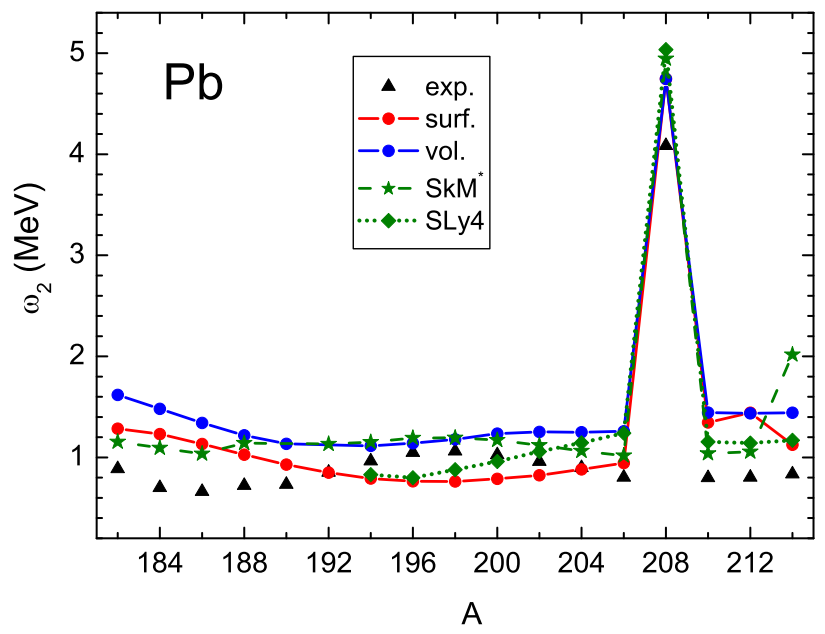

Fig. 6. Excitation energies $\omega\left(2_{1}^{+}\right)$for lead isotopes.

cantly higher than in neighboring even nickel isotopes that is a typical feature of magic nuclei. Although the effect is not so strong as for ${ }^{56} \mathrm{Ni}$, it is also rather pronounced. For a complete magicity, the distance between occupied singleparticle levels and the next free one should be greater than the double average gap value $2 \bar{\Delta}$. In this case, the gap equation has only the trivial solution. In the case under discus-

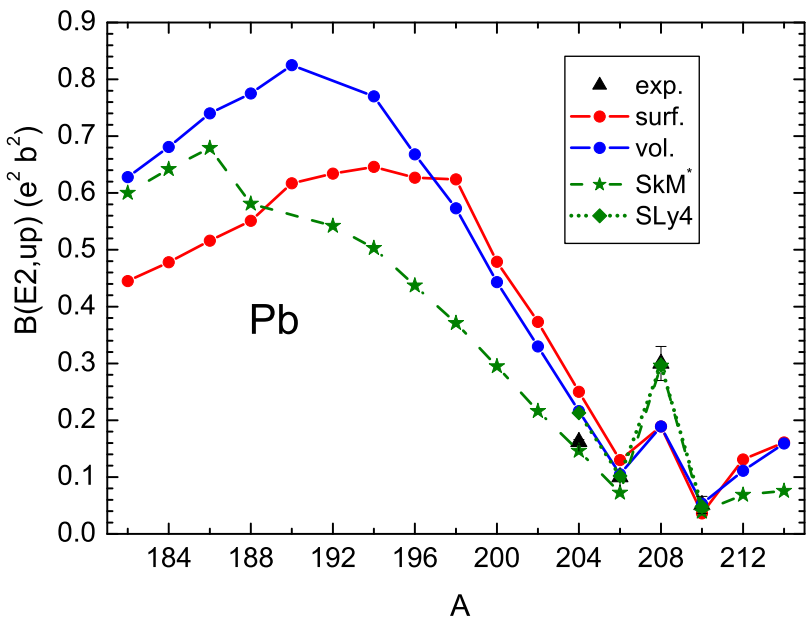

Fig. 7. $\mathrm{B}(E 2$, up) values for lead isotopes.

sion, the distance between the occupied $2 p-1 f$-shell and the next level $1 g_{9 / 2}$ is of the order of $2 \bar{\Delta}$ and the gap equation has a non-trivial solution. However, some features of magic nuclei occur in this nucleus but in a smoothed form. Thus, just as for the tin and lead chain, for analyzing the "surface versus volume" effect under discussion, it is worth to exclude the neighboring to ${ }^{56} \mathrm{Ni}$ and ${ }^{78} \mathrm{Ni}$ nuclei. In ad- 


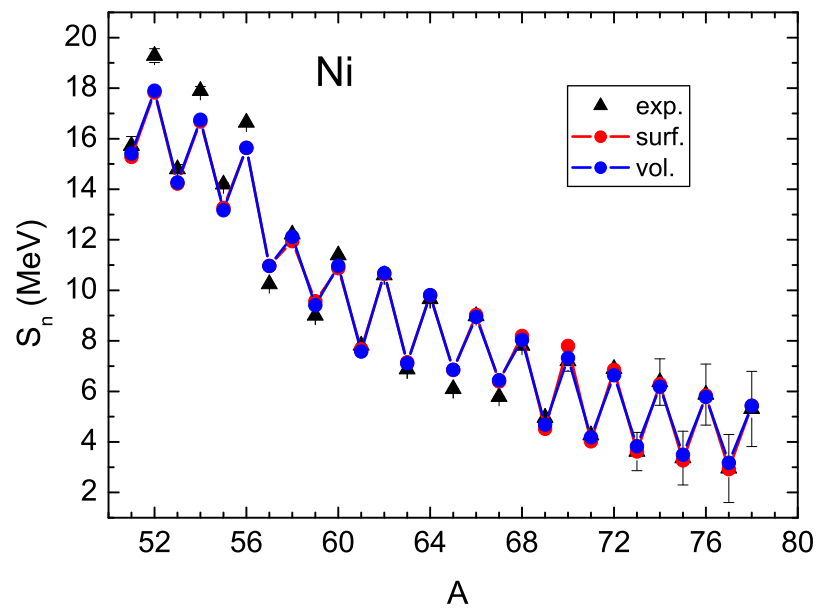

Fig. 8. Neutron separation energies $S_{n}$ for Ni isotopes.

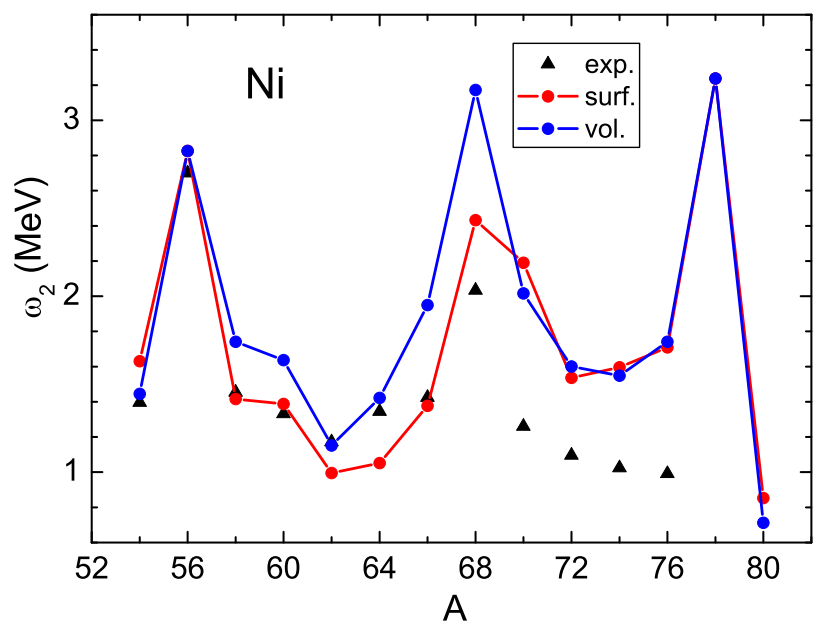

Fig. 9. Excitation energies $\omega\left(2_{1}^{+}\right)$for $\mathrm{Ni}$ isotopes.

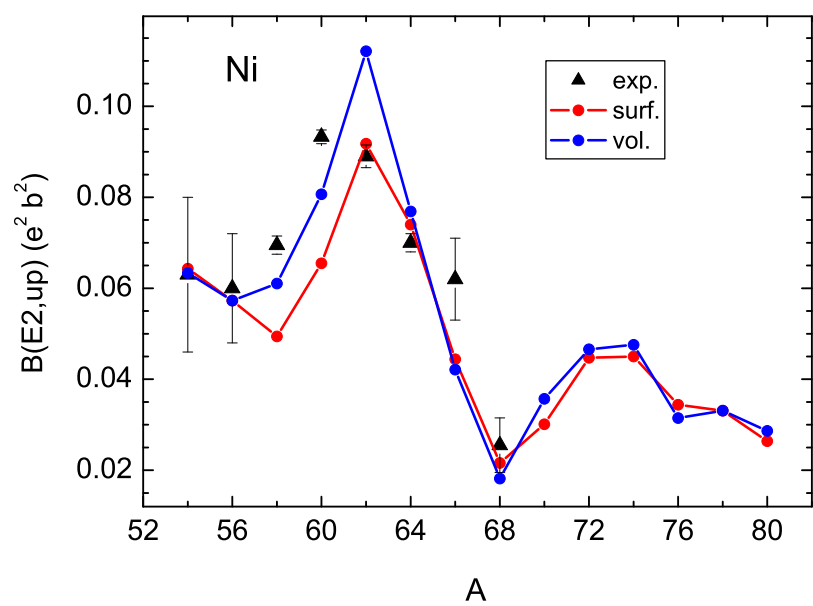

Fig. 10. $B(E 2$, up) values for $\mathrm{Ni}$ isotopes

dition, the isotopes ${ }^{66-70} \mathrm{Ni}$ should be also considered with some caution.

We see that qualitatively the pattern is similar to that in the tin and lead chains, however the effect for $\omega_{2}$ value is now not so regular. Indeed, for the isotopes ${ }^{72,74} \mathrm{Ni}$ it is practically zero and for the isotope ${ }^{70} \mathrm{Ni}$ it is of the opposite sign, although rather small. Although the latter could be interpreted as the effect of closeness to the quasi-magic nucleus ${ }^{68} \mathrm{Ni}$, let us try to understand the reason of such

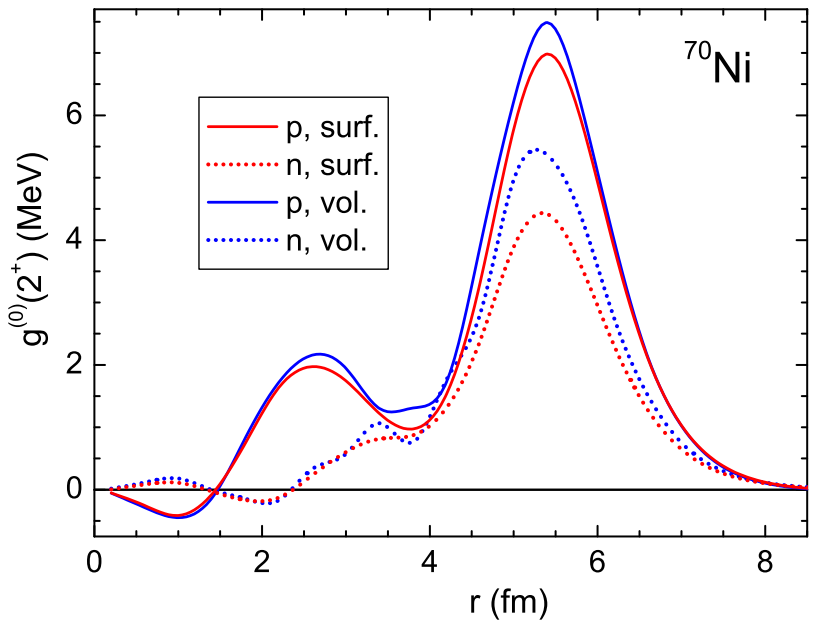

Fig. 11. The proton and neutron normal transition amplitude $g^{(0)}$ in the ${ }^{70} \mathrm{Ni}$ isotope.

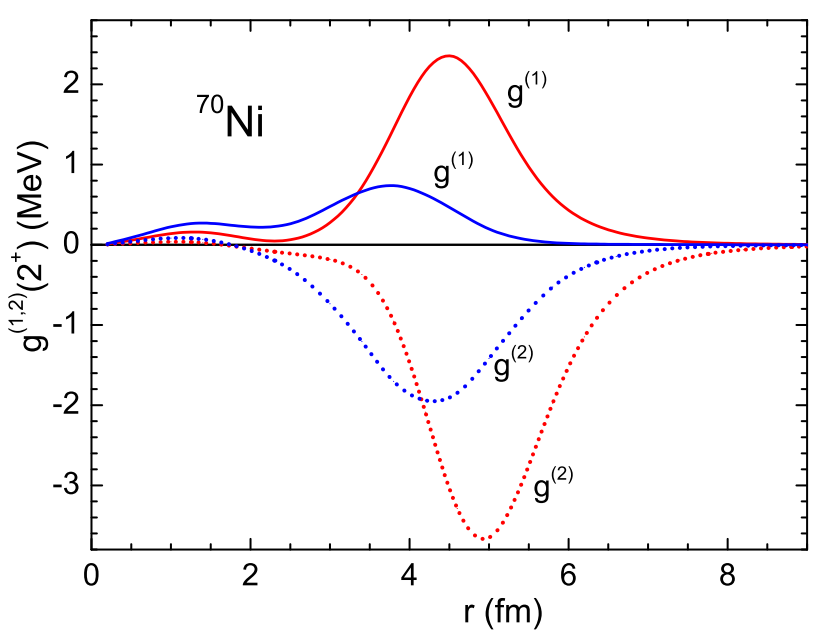

Fig. 12. The neutron anomalous transition amplitudes $g^{(1)}$ and $g^{(2)}$ in the ${ }^{70} \mathrm{Ni}$ isotope. Red solid and dotted lines correspond to surface pairing, blue ones, to volume pairing.

contradiction to the formula (12) within the scheme we use. For this aim, we displayed for this nucleus the normal and anomalous transition amplitudes in figure 11 and figure 12 correspondingly. We see that, just as for the ${ }^{118} \mathrm{Sn}$ nucleus, the normal amplitudes for the two models of pairing under consideration are very close to each other. In addition, again the anomalous amplitudes for the surface pairing case are much stronger than those for the volume pairing. However, in this nucleus the absolute values of the anomalous amplitudes are rather close to those of the normal amplitudes. Therefore, the consideration of the anomalous amplitudes as a small perturbation, which is the base for receiving the estimation (12), is not valid now.

Agreement with the data on $\omega_{2}$ values for the surface pairing case is almost perfect for nuclei lighter than ${ }^{68} \mathrm{Ni}$, however it becomes worse for heavier isotopes. The most strong disagreement for ${ }^{70} \mathrm{Ni}$ and ${ }^{76} \mathrm{Ni}$ nuclei again can be interpreted as the effect of closeness to magic nuclei (a quasi-magic in the first case). One more reason exists for poorer agreement for heavy nickel isotopes. For them, neutrons are filling up an almost isolated level $1 g_{9 / 2}$. Indeed, as it was already discussed, the distance to the filled levels of negative parity is about $2 \bar{\Delta}$ whereas that to neigh- 
boring levels of positive parity is significantly greater than $2 \bar{\Delta}$. In such a situation, predictions for the positive parity states depend significantly on details of the calculation scheme. The experimental values of transition probabilities are known only for isotopes till ${ }^{68} \mathrm{Ni}$, here agreement is quite reasonable.

\section{Conclusion}

Excitation energies, transition probabilities and other characteristics of the first $2^{+}$excitations in three chains of semimagic isotopes, the lead, tin and nickel ones, are calculated within the self-consistent TFFS on the base of the EDF by Fayans et al. [6]. The DF3-a functional [10] is used which differs from the original one DF3 with the spin-orbit and effective tensor terms only. A reasonable agreement with available experimental data is obtained. Predictions for other isotopes are made.

The effect of the density dependence of the effective pairing interaction is analyzed by comparing predictions of two models of the effective pairing interaction, the surface pairing and the volume one. The effect is found to be noticeable, especially for the $2^{+}$-energies which are, with the only exception, systematically higher at $200-300 \mathrm{keV}$ for the volume paring as compared with the surface pairing case. Thereby predictions of the surface pairing model agree with the data better than those of the volume model. Thus, the analysis of the excitation energies of the first $2^{+}$states in semi-magic nuclei gives a new evidence in favor of the surface pairing. As to $\mathrm{B}(E 2)$ values in tin isotopes, the volume pairing model looked better in comparison with old experimental data, but the new data of [16] agree better with the surface model.

\section{Acknowledgment}

We thank N. Pietralla for valuable discussion and information about new data on $\mathrm{B}(E 2)$ values in tin isotopes. The work was partly supported by the DFG and RFBR Grants Nos.436RUS113/994/0-1 and 09-02-91352NNIOa, by the Grants NSh-7235.2010.2 and 2.1.1/4540 of the Russian Ministry for Science and Education, and by the RFBR grants 11-02-00467-a and 12-02-00955-a.

\section{References}

1. S. T. Belyaev, Mat. Fys. Medd. Dan. Vid. Selsk. 32, No. 12 (1960).

2. S. V. Tolokonnikov, S. Kamerdzhiev, D. Voytenkov, S. Krewald, and E. E. Saperstein, Phys. Rev. C 84, 064324 (2011)

3. E. E. Saperstein and M. A. Troitsky, Sov. J. Nucl. Phys. 1, 400 (1965)

4. A. B. Migdal, Theory of finite Fermi systems and applications to atomic nuclei (Nauka, Moscow, 1965; transl. Wiley, New York, 1967)

5. M. V. Zverev and E. E. Saperstein, Sov. J. Nucl. Phys. 42, 400 (1985)

6. S. A. Fayans, S. V. Tolokonnikov, E. L. Trykov, and D. Zawischa, Nucl. Phys. A 676, 49 (2000)
7. S. S. Pankratov, M. V. Zverev, M. Baldo, U. Lombardo, and E. E. Saperstein, Phys. Rev. C 84, 014321 (2011)

8. V. A. Khodel and E. E. Saperstein, Phys. Rep. 92, 183 (1982)

9. W. Kohn and L. J. Sham, Phys. Rev. 140, A1133 (1965)

10. S. V. Tolokonnikov and E .E. Saperstein, Phys. Atom. Nucl. 73, 1684 (2010)

11. D. Voytenkov, S. Kamerdzhiev, S. Krewald, E. E. Saperstein, and S. V. Tolokonnikov, Phys. Rev. C 85, 054319 (2012)

12. A. Mukherjee, Y. Alhassid, and G. F. Bertsch, Phys. Rev. C 83, 014319 (2011)

13. J. E. Wise, et al., Phys. Rev. C 45, 2701 (1992)

14. V. A. Khodel, JETP Lett. 18, 72 (1973)

15. S. Raman, C. W. Nestor Jr., and P. Tikkanen, Atom. Data Nucl. Data Tables 78, 1 (2001)

16. A. Jungclaus, et al., Phys. Lett. B 695, 110 (2011)

17. J. Terasaki, J. Engel, and G. F. Bertsch, Phys. Rev. C 78, 044311 (2008)

18. D. C. Radford, et al., Nucl. Phys. A 752, 264c (2005)

19. J. Cederkäll, et al., Phys. Rev. Lett. 98, 172501 (2007)

20. C. Vaman, et al., Phys. Rev. Lett. 99, 162501 (2007)

21. A. Ekström, et al., Phys. Rev. Lett. 101, 012502 (2008)

22. M. East, et al., Phys. Lett. B 665, 147 (2008) 